\title{
Induction of Osteogenic Differentiation in Human Mesenchymal Stem Cells by Crosstalk with Osteoblasts
}

Martina Glueck, ${ }^{1,2}$ Oliver Gardner, ${ }^{1,3}$ Ewa Czekanska, ${ }^{1,3}$ Mauro Alini, ${ }^{1}$ Martin J. Stoddart, ${ }^{1}$ Gian M. Salzmann, ${ }^{2}$ and Hagen Schmal ${ }^{2, *}$

\begin{abstract}
Natural bone healing following fractures is initiated by osteoblasts (OBs) and mesenchymal stem cells (MSCs), a cell combination with possible potential in tissue engineering techniques for bony defects. The aim of the study was to investigate MSC/OB-crosstalk, in order to determine optimal cell culture conditions for osteogenic differentiation. Human OBs and MSCs interactions were investigated in an in vitro trans-well co-culture study over a time period of 28 days. Calcification was determined by optical density (OD) at $450 \mathrm{~nm}$ and Alizarin red staining. Messenger RNA expression was assessed by quantitative PCR. Osteogenic medium containing $1 \%$ fetal bovine serum resulted in superior levels of calcification in MSCs in co-culture with OBs compared to $2 \%$ or $5 \%$ fetal bovine serum $(p<0.05)$. Comparing MSCs and OBs alone with the MSC/OB co-culture, calcification, as measured by OD $450 \mathrm{~nm}$, increased over time in all groups. The highest values were recorded in the co-culture $(p<0.05)$ Osteogenic differentiation potential showed significant interindividual differences. In order to predict differentiation potential, OD $450 \mathrm{~nm}$ measurements and mRNA expression of alkaline phosphatase were correlated with the population doubling rate during the expansion period. For OBs and MSCs, statistically significant associations of proliferation and differentiation potential were found $(p<0.001)$. The addition of transforming growth factor beta resulted in up-regulation of collagen type I and Sp7 mRNA, and down-regulation of alkaline phosphatase mRNA. The results suggest the idea of soluble paracrine factors being secreted by OBs to induce osteogenic differentiation of MSCs.
\end{abstract}

Key words: mesenchymal stem cell; osteoblast; co-culture; osteogenic differentiation; bone formation

\section{Introduction}

Isolated bone or osteochondral defects in joints are seen following osteochondritis dissecans or traumatic injuries and are associated with a significant risk for development of consecutive osteoarthritis or non-unions. Therefore, these lesions need to be addressed by surgical repair. ${ }^{1}$ The multipotent nature of mesenchymal stem cells (MSCs), ${ }^{2}$ makes them an ideal candidate for cell therapy for musculoskeletal applications in- cluding bone regeneration. This is supported by their immune-suppressive regulatory properties, ${ }^{3}$ a characteristic that might be useful in cases with activation of inflammatory responses following acute impacts or fracture repair. Another theoretical advantage is the described higher tolerance of hypoxia compared with fully differentiated osteoblasts, allowing more time for the formation of a vascular network to supply the bone component. ${ }^{4}$ Knowledge about MSC differentiation

\footnotetext{
${ }^{1}$ AO Research Institute Davos, Davos Platz, Switzerland.

${ }^{2}$ Department of Orthopaedic and Trauma Surgery, University Medical Center, Albert-Ludwigs University Freiburg, Germany.

${ }^{3}$ Cardiff University, Cardiff, Wales, United Kingdom.
}

*Address correspondence to: Hagen Schmal, MD, University of Freiburg Medical Center, Department of Orthopaedic and Trauma Surgery, Hugstetter Street 55, Freiburg, D-79106, Germany, E-mail: hagen.schmal@freenet.de

(c) Martina Glueck et al. 2015; Published by Mary Ann Liebert, Inc. This Open Access article is distributed under the terms of the Creative Commons License (http://creativecommons.org/licenses/by/4.0), which permits unrestricted use, distribution, and reproduction in any medium, provided the original work is properly credited. 
in the vicinity of differentiated osteoblasts will also help to understand how natural repair of bony defects might function. Differentiation of MSCs may be controlled by different stimuli in cell culture media. ${ }^{2}$ Transforming growth factor beta (TGF $\beta$ ) is a key component regulating osteochondral differentiation and is prevalent during fracture repair. Dexamethasone/ $\beta$-glycerolphosphate have been shown to be required for osteogenic differentiation. ${ }^{5}$ A recently described opportunity for induction of an osteogenic phenotype ${ }^{6,7}$ is the co-culture with differentiated cells, a phenomenon that possibly plays an important role for the formation of the bone marrow niche. Here both cell-cell contacts and paracrine secretion mechanisms appear to play a significant role. ${ }^{6-8}$

The intention of this project was the definition of conditions that may support bone formation in a coculture of MSC and human osteoblasts (OBs). The influence of serum concentrations, cell functionality, and the presence of TGF $\beta$ were all investigated.

\section{Material and Methods}

Isolation of OBs and MSCs

Femoral heads were obtained during hip arthroplasty operations following femoral neck fractures. The material used was obtained with informed consent from patients in accordance with the University of Freiburg Medical Center Ethics Committee (Tissue Bank for Research in the Field of Tissue Engineering project [GTE-2002]). The degrees of osteoarthritis were evaluated on X-rays using Croft's modification of the Kellgren and Lawrence grading system. Cells from patients with advanced osteoarthritis ( $\geq$ grade 3 ) were not used for the experiments. Within 8 hours post-surgery osteoblasts [OBs] and mesenchymal stem cells [MSCs] were separated from one femoral head as previously described. ${ }^{9}$

Human primary osteoblasts were isolated from cancellous bone of the femoral heads by cell outgrowth from small pieces. ${ }^{10}$ MSCs were extracted from the bone marrow of cancellous bone of the same femoral heads. ${ }^{5}$ Briefly, for MSC isolation small pieces of cancellous bone were transferred in a conical tube with medium and vortexed. This step was repeated four times. Afterwards, mononuclear cells were separated by a Ficoll-Paque gradient (Pharmacia, Piscataway, $\mathrm{NJ}$ ), washed and seeded in flasks. For OB isolation, cells were additionally released from bone pieces by a short trypsin/EDTA digestion and seeded in flasks without further enrichment. Cells were aliquoted and stored frozen in liquid nitrogen until experiments were performed. The time from rethawing until the beginning of the experiment is referred to as the expansion period and differed among donors between 1 and 3 weeks due to differences in proliferation rates. MSCs were thawed 7-10 days later than the OBs due do the more rapid proliferation rate. During the expansion period, MSCs were cultured in Alpha Minimum Essential Medium (Gibco, Carlsbad, CA), 10\% fetal bovine serum (FBS) (MSC qualified, Gibco), $5 \mathrm{ng} / \mathrm{mL}$ FGF-2 (Invitrogen, Karlsruhe, Germany), 1\% penicillin/streptomycin. OBs were cultured in Dulbecco's modified Eagle's medium (DMEM)/ low glucose (1g/L, Gibco), $10 \%$ FBS (heat inactivated, Gibco) and 1\% penicillin/ streptomycin.

\section{Experimental setup}

MSCs in the bottom and OBs on top were separated in a trans-well culture with $0.4 \mu \mathrm{m}$ inserts. As a basal culture medium DMEM/low glucose ( $1 \mathrm{~g} / \mathrm{L}$, Gibco) without phenol-red, supplemented with ascorbic acid $50 \mu \mathrm{g} / \mathrm{mL}, \beta$-glycerolphosphate $5 \mathrm{mM}$, dexamethasone $1 \times 10^{-7} \mathrm{M}$, nonessential amino acids $1 \%$, ITS $1 \%$, HEPES buffer $20 \mathrm{mM}$, and penicillin/streptomycin $1 \%$ was used. Half media changes were performed three times per week. The initial seeding density was $20,000 \mathrm{cells} / \mathrm{cm}^{2}$. The cells from the different donors were combined as indicated in Table 1 and were used at a maximum passage of 4 . The total time of co-culture was 28 days.

The effect of adding FBS to the basal culture medium in increasing concentrations of $1 \%, 2 \%$, and $5 \%$ was tested. In order to examine the effect of TGF $\beta$, the medium was additionally supplemented with $2 \mathrm{ng} / \mathrm{mL}$ TGF $\beta$-1 (Invitrogen) during the whole culture period.

\section{Quantitation of osteogenic differentiation}

Calcification was assessed using two independent methods. At first, a spectroscopic analysis was done measuring the absorbance (optical density) at $450 \mathrm{~nm}$ to allow for nondestructive online measurement. Calcium and phosphate deposition marking the beginning

Table 1. Cell Combinations Used in Each Run of the Experiment

\begin{tabular}{|c|c|c|c|}
\hline & Experiment run 1 & Experiment run 2 & Experiment run 3 \\
\hline MSC & 9 , age 73 years & $\$$, age 83 years & ๙, age 76 years \\
\hline $\mathrm{OB}$ & o, age 76 years & $\delta$, age 72 years & q, age 73 years \\
\hline
\end{tabular}


Table 2. Overview of Primers and Probes Used

\begin{tabular}{|c|c|c|c|}
\hline Gene & Primer forward $\left(5^{\prime}-3^{\prime}\right)$ & Primer reverse $\left(5^{\prime}-3^{\prime}\right)$ & Probe (5'FAM/3'TAMRA) \\
\hline 18S rRNA & \multicolumn{3}{|c|}{ rRNA endogenous control, VIC/TAMRA, 4310893E-1105050, X03205.1 } \\
\hline ALP & \multicolumn{3}{|c|}{ Assay on demand, FAM, Hs00758162_m1, PN4351370 } \\
\hline COL1A1 & CCC TGG AAA GAA TGG AGA TGA T & ACT GAA ACC TCT GTG TCC CTT CA & CGGGCAATCCTCGAGCACCCT \\
\hline Sp7 & CCT GCT TGA GGA GGA AGT TCA & GGC TAG AGC CAC CAA ATT TGC & TCC CCT GGC CAT GCT GAC GG \\
\hline
\end{tabular}

ALP, alkaline phosphatase; COL1A1, collagen type 1 .

of the bone formation process, in combination with the increasing cell density, leading to a higher absorbance. $^{11}$ Measurements were taken at day $7,14,21$, and 28 from the cells within the wells. Secondly, the more specific Alizarin red staining (ARS) of the cells within the wells at day 28 and a subsequent quantitative assay were done. Alizarin red working solution (SigmaAldrich, St. Louis, MO) was prepared to a concentration of $40 \mathrm{mM}$ and a $\mathrm{pH}$ of $4.1-4.3$. After 28 days of culture the samples were fixed with $4 \%$ formaldehyde, washed, incubated with the dye for $30 \mathrm{~min}$ on a rotating plate, and then washed with double distilled water. For quantification, the stained monolayer was dissolved in $10 \%$ acidic acid. The suspension was heated to $85^{\circ} \mathrm{C}$ for $10 \mathrm{~min}$ and centrifuged at $20,000 \mathrm{~g}$ for $15 \mathrm{~min}$ after cooling on ice. The supernatant was collected and $\mathrm{pH}$ was adjusted to 4.2 with ammonium hydroxide. For quantification, the samples' absorption at an optical density (OD) of $405 \mathrm{~nm}$ was plotted against a standard.

The population doubling rate (PDR) was used to compare proliferation capacity among cells of different donors. PDR was calculated for the pre-culture expansion time from thawing until seeding the cells using the formula: $3.32 \times($ LOG [cell count last day of preculture]-LOG[cell count first day of preculture]) divided by the day number of preculture period.

\section{Quantitative PCR}

Gene up-regulation by day 28 compared to day 0 (seeding day) was analyzed by RT-PCR using $18 \mathrm{~S}$ rRNA as a housekeeping gene. At day 0 and 28 approximately $1 \times 10^{6}$ OBs and MSCs were taken for separate RNA isolation. The cell pellets were resuspended in $1 \mathrm{~mL}$ TRI Reagent (MRC Global Inc., Houston, TX) and $5 \mu \mathrm{L}$ of polyacryl carrier (MRC Global Inc.) was added. At day 28 the top cells growing in the co-culture insert were washed, dissolved in $300 \mu \mathrm{L}$ TRI Reagent and $5 \mu \mathrm{L}$ of polyacryl carrier was added. Alteration of gene expression was measured on the MSCs cocultured with OBs (MSC_OB). MSCs co-cultured with MSCs (MSC_MSC) were used as negative control whereas OBs co-cultured with OBs (OB_OB) served as positive control. RNA was isolated by phase separation with 1-Bromo-3-chloropropane (Sigma-Aldrich) and several subsequent washing steps. For reverse transcription, the TaqMan Reverse Transcription Reagent kit (Invitrogen) with $1 \mu \mathrm{g}$ total RNA was used with random oligomers as per manufactures instructions. For PCR, TaqMan Gene Expression Master Mix (Applied Biosystems, Carlsbad, CA) and human primers and probes (all from Applied Biosystems) with the sequences shown in Table 2 were used.

\section{Statistical analysis}

All values of one experiment were expressed as mean \pm standard deviation and each experiment was repeated three times. Individual group medians were compared with the Mann-Whitney U rank sum test. For multiple comparisons, post hoc statistic (KruskalWallis H-test) was used to analyze statistical significances between the grouped values. Correlations were determined by calculating the Spearmen coefficient $(\rho)$; the coefficient of determination $\left(\mathrm{R}^{2}\right)$ was calculated by means of a regression analysis. Statistical significance was defined when $p<0.05$.

\section{Results}

Influence of serum content on osteogenic differentiation

The effect of serum concentration in a trans-well coculture for MSCs in the bottom (b) with OBs loaded on top ( $t$ ) was examined. A spectroscopic analysis measuring the absorbance at $450 \mathrm{~nm}$ was used in order to monitor the calcification. After 28 days for all three donors, the highest degree of calcium deposition was found using $1 \%$ FBS (heat inactivated, $0.36 \pm 0.06$, donor 2) added to the culture medium compared to $2 \%(0.30 \pm 0.16$, donor 2$)$ and $5 \%(0.10 \pm 0.04$, donor 2). Statistical significances were found as indicated in Figure 1. Although the effect of FBS concentration was reproducible using cells from different donors, 


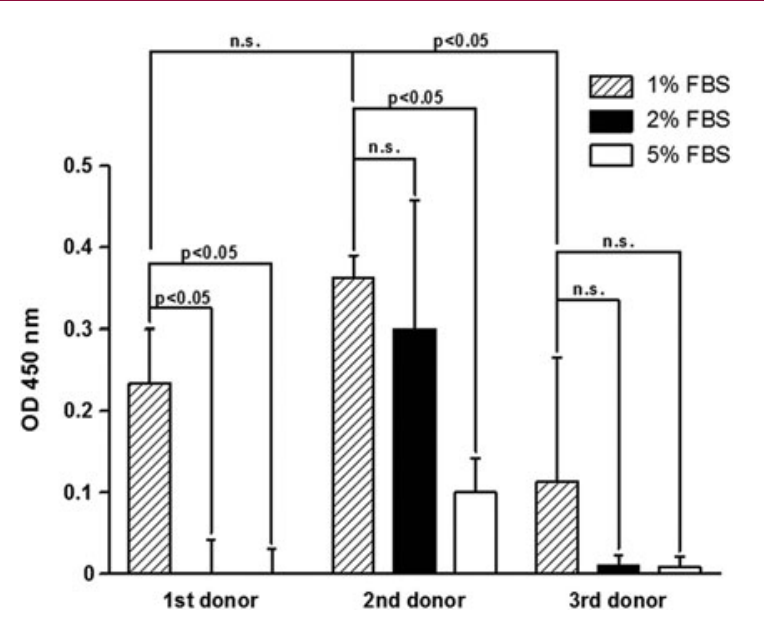

FIG. 1. The figure shows the effect of serum concentration in a trans-well co-culture for mesenchymal stem cells (MSCs) in the bottom with osteoblasts (OBs) loaded on top. A spectroscopic analysis measuring the absorbance at $450 \mathrm{~nm}$ was used in order to quantitate the calcification. After 28 days for all three donors, the highest degree of calcium deposition was found using $1 \%$ fetal bovine serum (FBS) added to the culture medium. OD, optical density, n.s., not significant.

there also were remarkable differences between the donors. Optical density measured for the cultures with $1 \%$ FBS reached the highest absorbance in donor 2 followed by donor $1(0.23 \pm 0.06, \mathrm{p}=0.248)$ and donor $3(0.11 \pm 0.15, p=0.0495)$. For the $2 \%$ concentration, the measurements reached statistical significance for all donors.

An increasing OD at $450 \mathrm{~nm}$ over the 4 weeks culture period could be observed in all groups [MSCs alone as negative control, OBs alone as positive control, co-culture of MSCs in the bottom (b) with MSCs loaded on top (t), MSC(b)_MSC(t); co-culture of OBs in the bottom (b) with OBs loaded on top ( $t$ ), $\mathrm{OB}(\mathrm{b}) \_\mathrm{OB}(\mathrm{t})$; co-culture of MSCs in the bottom (b) with OBs loaded on top (t), MSC(b)_OB (t)] as shown in Fig. 2 (representative experiment, KruskalWallis $\mathrm{H}$-Test, $p<0.025$ for all groups).

MSCs in co-culture with OBs showed the highest absolute value of extinction at $450 \mathrm{~nm}$ by day $28 \mathrm{com}-$ pared with all other groups $(0.36 \pm 0.03, p=0.049)$. This maximum of $\mathrm{OD}$ at $450 \mathrm{~nm}$ was followed by OBs alone (0.22 \pm 0.07$) ; \mathrm{OB}(\mathrm{b}) \_\mathrm{OB}(\mathrm{t})(0.21 \pm 0.06)$;

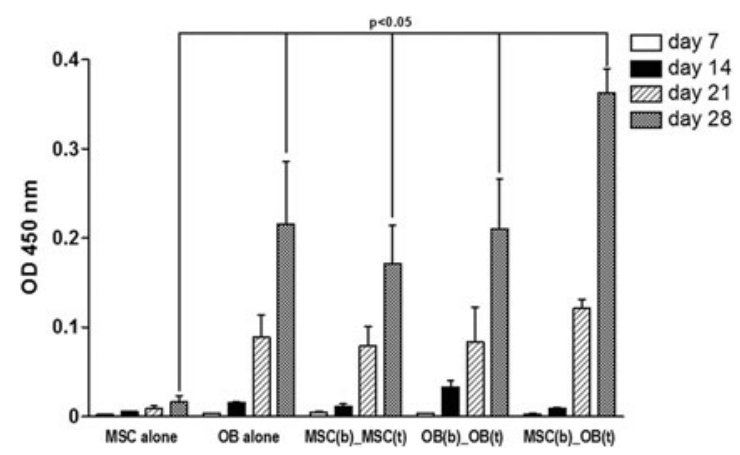

FIG. 2. An increasing OD at $450 \mathrm{~nm}$ over the 4-week culture period could be observed in all groups [Kruskal-Wallis H-test $p<0.025,1 \% \mathrm{FBS}$ ) (MSCs alone as negative control; OBs alone as positive control, co-culture of MSCs in the bottom (b) with MSCs loaded on top (t), MSC(b)_MSC(t); co-culture of $\mathrm{OBs}$ in the bottom (b) with $\mathrm{OBs}$ loaded on top (t), $\mathrm{OB}(\mathrm{b}) \_\mathrm{OB}(\mathrm{t})$; co-culture of MSCs in the bottom (b) with OBs loaded on top (t), MSC(b)_OB (t)]. MSCs in co-culture with OBs showed the highest absolute value of extinction at $450 \mathrm{~nm}$ by day 28 compared with all other groups $(p=0.049)$.

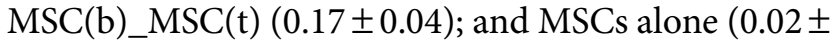
$0.01)$. Data show the remarkable effect of the co-culture by itself on the osteogenic differentiation of MSCs, comparing the indicated values for the extinction by MSCs alone and MSC(b)_MSC(t). At day $14 \mathrm{OD}$ at $450 \mathrm{~nm}$ was still higher in the co-culture of OBs compared to MSCs, but after this time point extinction values equaled each other. This observation was independent of serum concentration and donor.

The data of OD $450 \mathrm{~nm}$ measurements correlates with ARS of cultures within the culture well (Fig. $3 \mathrm{~A}, \mathrm{~B})$. This was confirmed by the subsequent quantitative assay at day 28 showing the highest absorbance and amount of Alizarin red dye for the coculture containing $1 \%$ FBS $(17.0 \pm 19.3 \mu \mathrm{M})$ compared with $2 \%(11.4 \pm 19.4 \mu \mathrm{M})$ and $5 \% \quad$ FBS $(4.6 \pm 5.5 \mu \mathrm{M})$ (Fig. 3A). Data only mark a trend, as no statistically significant differences between the groups were observed. Interestingly, there was a difference between the $\mathrm{MSC} / \mathrm{OB}$ co-culture and the MSC control, because spontaneous calcification of MSC alone was higher using $2 \%$ serum concentrations 


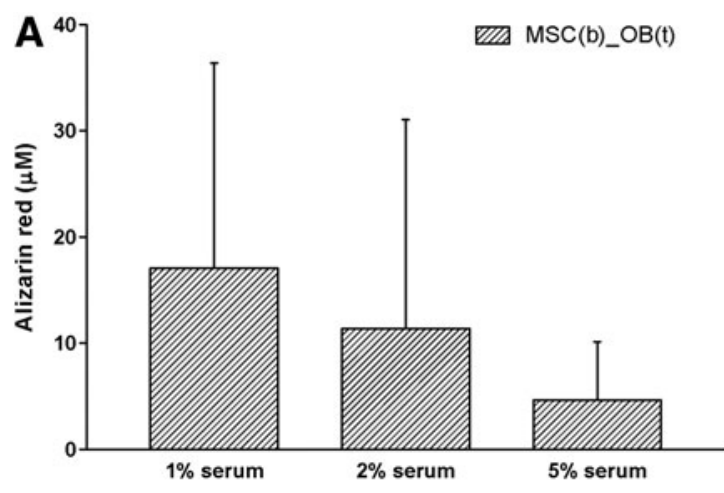

B
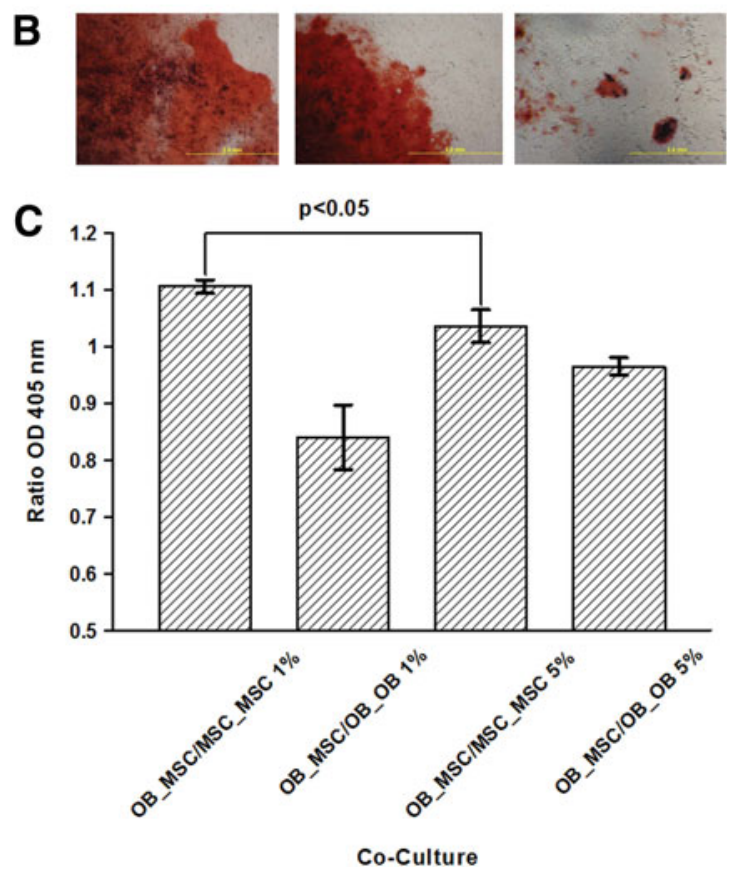

FIG. 3. Microscopy at day 28 for Alizarin red staining (A) of MSCs in the bottom wells of a coculture with $\mathrm{OBs}$ on top showing increasing calcification with decreasing serum concentration. This was confirmed by the subsequent quantitative assay at day 28 (B). A new calculation based on individual ratios was added in order to eliminate the variations between the different donors. Correlating the OD 450 data, this resulted in a statistically significant higher intensity of Alizarin red staining of the co-culture (OB_MSC) using $1 \%$ serum compared to $5 \%$ (C).
$(0.00 \pm 0.00 \mu \mathrm{M} \quad$ vs. $2.35 \pm 4.10 \mu \mathrm{M})$. This analysis resulted in very high standard deviations caused by marked interindividual differences. Therefore, we carried out calculations based on the ratios of our investigation group (co-culture of OB and MSC [OB_MSC]) with the negative control (co-culture of MSC and MSC [MSC_MSC]) and the positive control (co-culture of OB and OB [OB_OB]) calculated for each individual. Based on this calculation, ARS was higher in the $1 \%$ group $(1.1 \pm 0.01)$ compared with the $5 \%$ group $(0.84 \pm 0.06, p<0.05$, Fig. $3 C)$. Furthermore, the difference between positive and negative control ratios was also statistically significant different for the $1 \%$ and the $5 \%$ group $(p<0.05)$. Even the positive control of the $5 \%$ group showed statistically significant less calcification (ARS) than the positive control ratio of the $1 \%$ group.

\section{Influence of cell function}

on osteogenic differentiation

Although the principal effects of serum concentration on calcification were the same independent of the donors, a substantial variation of the interindividual absolute values was observed. This fact initiated the examination of a possible association of proliferation and differentiation potential. Therefore, osteogenic differentiation, represented by OD $450 \mathrm{~nm}$, was correlated with the number of population doublings per day during the expansion period. Data showed that fast growing OBs with a high PDR per day showed higher values of absorbance at $450 \mathrm{~nm}$ after 28 days of culture, indicating better osteogenic differentiation. In Fig. 4A the OB PDR for each donor was plotted against the OD $450 \mathrm{~nm}$. In the case of all three donors a strong positive correlation of PDR and the absorbance at $450 \mathrm{~nm}$ could be seen within the group of the same serum concentration $\left(\mathrm{R}^{2}\right.$ for $1 \%$ FBS $0.90 ; \mathrm{R}^{2}$ for $2 \%$ FBS $0.98 ; \mathrm{R}^{2}$ for $5 \%$ FBS 0.74$)$. Although the coefficients of determination indicated overall good associations, only in the groups with $1 \%$ and $2 \%$ FBS were the correlations significant $(p<0.0001)$. The regression curve for the $1 \%$ FBS group had the steepest slope, indicating greater calcium deposition. This is in line with the highest value of absorption at $450 \mathrm{~nm}$ compared to higher serum concentrations. This also correlates with the highest amount of calcification measured by Alizarin red staining in the MSC/OB co-culture as presented in Fig. 3. Overall, fast growing OBs seem to have a higher potential to calcify compared to slowly growing OBs. 

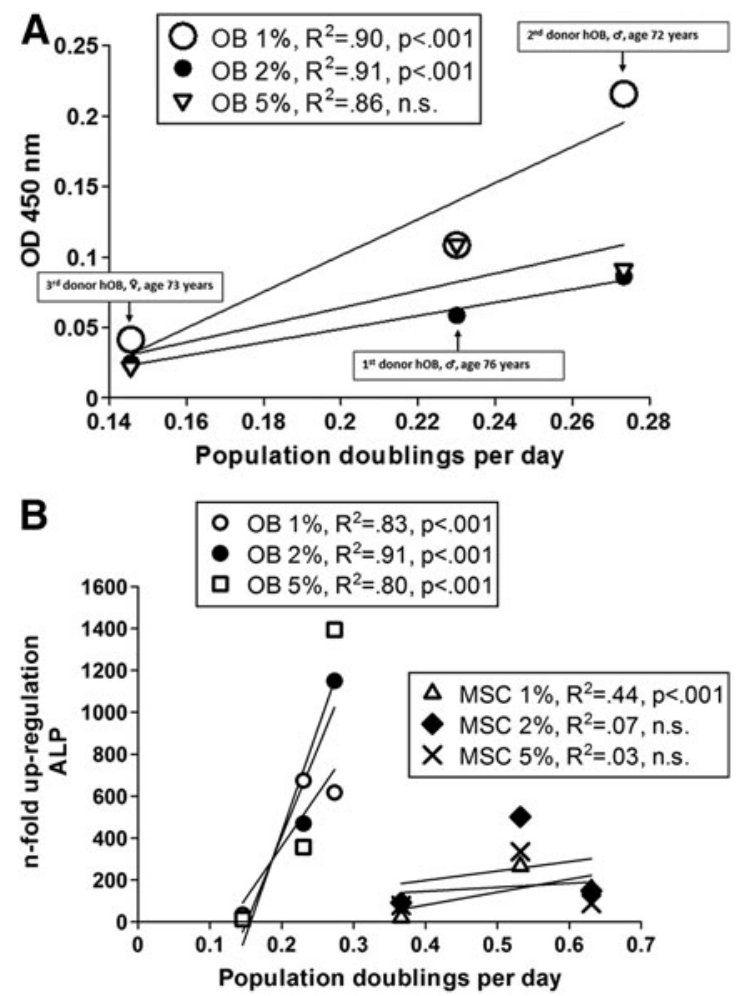

FIG. 4. (A) The association of the population doubling rate during the expansion period with the OD at $450 \mathrm{~nm}$ is shown, demonstrating that donor-dependent proliferation determines ability for osteogenic differentiation. This could be shown for all investigated serum concentrations as indicated in the legend. hOB, human osteoblasts. (B) The association of the population doubling rate during the expansion period with alkaline phosphatase (ALP) mRNA expression is shown, demonstrating that donor-dependent proliferation determines ability for osteogenic differentiation. This could be shown for all investigated serum concentrations and for both cell types (MSC right and OB left) as indicated in the legend.

The same positive correlation between population doublings within a group of the same serum concentration could be seen for up-regulation of alkaline phosphatase (ALP) mRNA in OBs within the 28 day cell culture period measured by quantitative PCR $\left(\mathrm{R}^{2}\right.$ for $1 \%$ FBS $0.83 ; \mathrm{R}^{2}$ for $2 \%$ FBS $0.91 ; \mathrm{R}^{2}$ for $5 \%$ FBS 0.80; Fig. 4B, left). The steepest slope was observed for the group with $5 \%$ FBS. The associations of all different groups reached statistical significance $(p<0.0001)$. ALP up-regulation is an indicator for osteogenic differentiation, which correlates with proliferation potential. Although the coefficients of determination were much lower for MSCs, a similar association could be demonstrated for this cell type (Figure 4B, right) reaching statistical significance for the $1 \%$ FBS concentration calculating the Spearman correlation.

\section{Influence of TGF $\beta$ on osteogenic differentiation}

The addition of TGF $\beta$ reduced the calcification in the co-culture of MSCs with OBs measured by the $450 \mathrm{~nm}$ absorbance (Fig. 5). This effect was not dependent on serum concentration. As seen previously, OD $450 \mathrm{~nm}$ was significantly higher $(p=0.0495)$ after 28 days using $1 \%$ FBS $(0.36 \pm 0.03)$ compared with $5 \%$ FBS $(0.10 \pm 0.04)$. OD was diminished by TGF $\beta$ to $0.12 \pm 0.02$ in the $1 \%$ FBS co-culture and to $0.014 \pm 0.001$ in the 5\% FBS co-culture $(p=0.0495)$. Similarly, Alizarin red staining was reduced in the TGF $\beta$ treated cultures without reaching statistical significance $(17.0 \pm 19.3 \mu \mathrm{M}$ vs. $13.0 \pm 11.3 \mu \mathrm{M})$.

Real time PCR demonstrated that TGF $\beta$ caused a statistically significant up-regulation of collagen type I mRNA in all co-cultures ( $n=4$ per co-culture, Fig. 6A). This effect was confirmed for both investigated

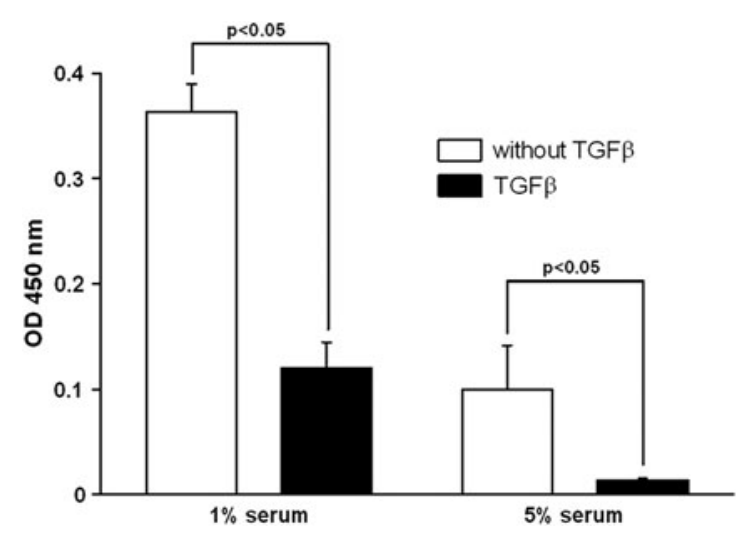

FIG. 5. This graph shows the effect of TGF $\beta$ on calcification of MSCs in the bottom of a co-culture with OBs on top mirrored by OD $450 \mathrm{~nm}$. Independent of serum concentration, a statistically significant inhibition of terminally osteogenic differentiation could be demonstrated ( $p=0.0495)$. TGF $\beta$ transforming growth factor beta. 

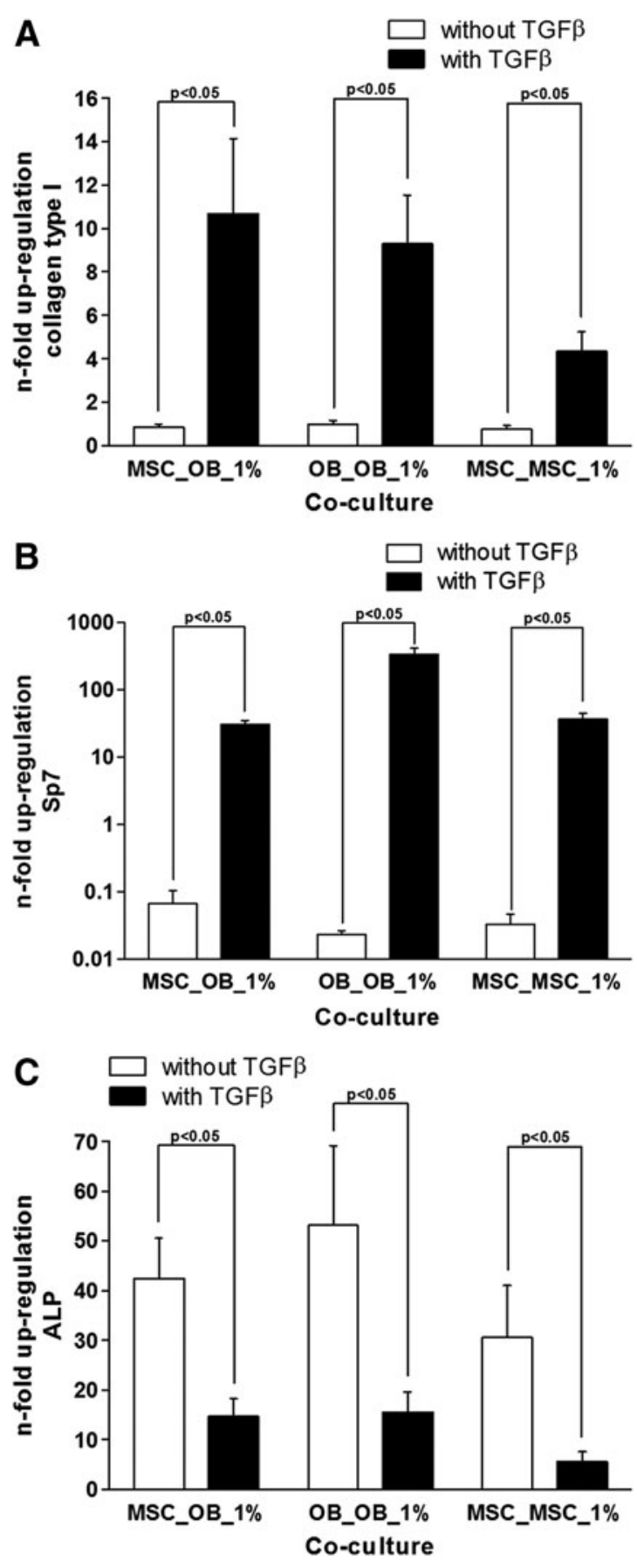

FIG. 6. The figure shows the influence of TGF $\beta$ on the expression of the genes for collagen type 1 (A), the transcription factor Sp7 (B), and alkaline phosphatase (C) in co-cultures of mesenchymal stem cells (MSC) with osteoblasts (OB) (MSC_OB); $\mathrm{OB}$ and $\mathrm{OB}$ (OB_OB); and MSC and MSC (MSC_MSC) in medium containing $1 \%$ FBS. Whereas collagen type 1 (col1a) and Sp7 were upregulated, expression of ALP was suppressed. cell types, MSCs and OBs. In contrast, collagen type 2 mRNA was not constitutively expressed by MSC or OB and could also not be found after the 28 days of coculture in osteogenically differentiated MSC or other cell types. Alteration of gene expression was measured in MSCs co-cultured with OBs (MSC_OB). MSCs cocultured with MSCs (MSC_MSC) served as the negative control, whereas OBs co-cultured with $\mathrm{OBs}$ (OB_OB) were regarded as the positive control. Data shown represent an experiment using 1\% FBS, but the effects were constantly seen using the other investigated FBS concentrations. Addition of TGF $\beta$ caused an up-regulation of collagen type 1 mRNA (collA1) in the MSC_OB co-culture $(2.78 \pm 0.33, p=0.021)$, the OB_OB co-culture $(9.29 \pm 2.25, p=0.021)$, and the MSC_MSC co-culture $(4.33 \pm 0.89, p=0.021)$ compared to the non-treated samples. The lowest collA1 expressions in the groups without TGF $\beta$ treatment were found in the MSC_OB co-culture $(0.46 \pm 0.14)$ followed by the MSC_MSC co-culture $(0.75 \pm 0.17)$ and the OB_OB co-culture $(0.97 \pm 0.18)$.

It further could be demonstrated that TGF $\beta$ caused a statistically significant up-regulation of Sp7 mRNA in all co-cultures ( $n=4$ per co-culture, Fig. $6 \mathrm{~B})$. Whereas Sp7 mRNA expression was almost completely downregulated after 28 days cell culture compared to day 0 without TGF $\beta$ (MSC_OB $0.07 \pm 0.04$, OB_OB $0.02 \pm 0.003$, MSC_MSC $0.03 \pm 0.01$ ), addition of TGF $\beta$ caused an up-regulation in the MSC_OB coculture $(30.86 \pm 3.94, \quad p=0.021)$, the OB_OB $(335.88 \pm 79.81, p=0.021)$, and the MSC_MSC co-culture $(37.20 \pm 7.32, p=0.021)$. There was no statistically significant difference between the different groups without TGF $\beta$ treatment. In the groups with TGF $\beta$ there was no statistically significant difference between the MSC_OB and the MSC_MSC co-culture, but there was a statistically significant difference between both groups and the OB_OB control $(p=0.021)$.

It also could demonstrated that TGF $\beta$ caused a statistically significant down-regulation of ALP mRNA in all co-cultures ( $n=4$ per co-culture, Fig. 6C). Whereas ALP mRNA expression showed a large upregulation after 28 days equally in all co-cultures, which is in line with the ongoing osteogenic differentiation (MSC_OB 25.92 \pm 7.29 , OB_OB 53.10 \pm 15.98 , MSC_MSC $30.56 \pm 10.35$ ), addition of TGF $\beta$ caused a down-regulation of ALP in the MSC_OB co-culture $(4.34 \pm 1.32, p=0.021)$, the OB_OB $(15.50 \pm 4.06$, $p=0.021)$, and the MSC_MSC co-culture (5.59 \pm 1.96, $p=0.021)$. The lowest ALP expressions in the 
groups without TGF $\beta$ treatment were found in the MSC_OB co-culture $(25.93 \pm 7.30)$, followed by the MSC_MSC co-culture $(30.57 \pm 10.34)$ and the OB_ OB co-culture $(53.10 \pm 15.99)$. In the groups with $\operatorname{TGF} \beta$ there was no statistically significant difference between the MSC_OB and the MSC_MSC co-culture, but there was a statistically significant difference between MSC_OB and the OB_OB control $(p=0.021)$.

Experiments investigating the mRNA expression of collA1, Sp7, and ALP were repeated three times, also applying the different serum concentrations $(2 \%$ and $5 \%)$, showing equal regulation patterns.

\section{Discussion}

The most important finding of this study was the induction of calcification in MSCs by a co-culture with OBs, which was highly dependent on serum concentration. In contrast to standardized animal experiments, human donors demonstrated high individual variances in their differentiation potential, which were predictable based on the population doubling rate. Furthermore, the influence of TGF $\beta$ was remarkable leading to the induction of early markers of osteogenesis as collagen type 1 and Sp7 and inhibition of indicators of the final steps of osteogenic differentiation shown by suppression of ALP.

Tissue engineering of bone with progenitor cells, such as adult mesenchymal stem cells (MSCs), appears to be a promising strategy for the treatment of bone defects. The technique of co-culture is a possible strategy, which may avoid the application of specific differentiation stimuli. In fact, osteogenic differentiation of progenitor cells induced by co-culture with differentiated osteoblasts has been previously demonstrated by other groups. ${ }^{6,7,12}$ Whereas some publications emphasize the importance of cell-cell interactions, ${ }^{7}$ others demonstrated a paracrine mechanism $^{6}$ as confirmed in the presented experiments. Although the principle of successful induction of an osteogenic phenotype in the progenitor cells by coculture with OBs was previously described, for tissue engineering applications it is absolutely necessary to define culture conditions retaining the most efficient results. This is also important considering a possible generation of osteochondral constructs by MSC, in which paracrine effects of neighboring OBs and chondrocytes might initiate the differentiation of a chondral or osteogenic layer. Serum conditions were found to significantly affect proliferation and differentiation capacity of human adipose stem cells. ${ }^{13}$ There- fore, the effect of serum concentration was evaluated, showing the highest amount of calcium deposition when using a low serum environment.

Considering application in humans, a pronounced interindividual variation of differentiation capacity has to be taken into account. This could be confirmed by our experiments together with the opportunity to predict the differentiation potential by simply determining the population doubling rate of MSCs or OBs during the expansion period. An association of proliferation capacity with osteogenic differentiation potential in adipose derived MSC has been suggested before in rabbits, pigs and rats, ${ }^{14}$ but in the case of human donors may simply reflect biological cell health. During an amplification process osteoblasts or chondrocytes usually loose some characteristics of their original differentiation, ${ }^{9}$ but may restore the phenotype under cell-specific culture conditions. The presented correlation of proliferation with differentiation capacity does not indicate that the cells exhibit the osteogenic properties during the whole amplification period, it just refers to the capacity to restore the specific phenotype.

The role of TGF $\beta$ in osteochondral differentiation has been described, showing that proliferation of MSCs and their differentiation into chondrocytes is stimulated, osteoblast progenitor's differentiation into osteoblast is promoted, and hematopoietic stem cells are kept in a state of hibernation. ${ }^{15}$ It has been shown that high concentrations of TGF $\beta$ enhance OBs proliferation and down-regulates the expression of receptor activator of nuclear factor kappa B ligand, whereas low concentrations enhance osteoclast maturation. ${ }^{15}$ Similar to the presented data, Schagemann et al. have found that the initial release of TGF $\beta$ induced an expression of type 1 collagen and osteogenic marker genes in MSCs. ${ }^{16}$ Considering the constant and low dose TGF $\beta$ stimulus used in this experimental setup, it may be concluded that early markers of ossification, collagen type 1 and Sp7, were induced and indicators of the final steps of osteogenic differentiation such as ALP were inhibited. This is supported by a recently published clinical trial showing a correlation of serum TGF $\beta$ values with the incidence of non-unions in humans. ${ }^{17} \mathrm{~A}$ similar regulation pattern was observed in BMP-4 transfected C3H10T1/2 cells, in which the ALP peak values coincided with down-regulation of type 1 collagen. ${ }^{18}$ This is in line with the finding that Sp7 is necessary, but not sufficient for human MSC differentiation into osteoblasts. ${ }^{19}$ 
MSCs co-cultured with OBs showed levels of calcification measured by absorption at $450 \mathrm{~nm}$ and Alizarin red staining comparable to the positive controls of OBs alone or the co-culture of OBs and OBs. Whereas MSCs alone demonstrated only very low levels of spontaneous calcification, the addition of MSCs to MSCs in a co-culture resulted in a remarkable increase of osteogenic characteristics. Contrary to these results, mRNA levels in 1\% FBS osteogenic medium did not show statistically significant differences in up- or down-regulation of the genes col1A1, Sp7, and ALP comparing the co-culture of MSCs and OBs with MSCs and MSCs. The discrepancy between calcification and gene expression might suggest that the OBs in the co-culture are mainly responsible for the calcification, possibly triggering by the secretion of soluble ALP. Since only the endpoint after 28 days has been analyzed, another explanation would be that ALP expression had already peaked earlier in the co-culture of MSCs with OBs as it has been described. ${ }^{20}$

Despite the fact that the data also add knowledge about natural repair mechanisms regarding osteogenesis, there are limitations caused by the study design. The experiments were all done in trans-well co-cultures using monolayers. The osteogenic differentiation cascade is of course influenced by a possible scaffold providing a three-dimensional environment, ${ }^{21}$ a factor, which was not addressed with the current experimental setup. Although the principal effects described could be observed independent of the donors, high interindividual discrepancies were observed. When interpreting the data, the limited number of cell donors also needs to be kept in mind. This issue is always relevant when working with human specimens, but may be addressed by the estimation of differentiation potential using the population doubling rate. Furthermore, the experimental design only allows the paracrine effects to be evaluated and neglects cell-cell interactions.

To summarize, the data of this study indicate that low serum conditions support osteogenesis and that TGF $\beta$ mediates the initial molecular steps but counteracts final differentiation. The interindividual variations in humans are high, but may be predicted to a certain degree by observing cell functions a proliferation capacity.

\section{Acknowledgments}

The study was funded by the Research Commission of the University Medical Center, Albert-Ludwigs Univer- sity Freiburg and the Department of Education and Research Germany (01EC1001D). The article processing charge was funded by the open access publication fund of the Albert Ludwigs University Freiburg.

\section{Author Disclosure Statement}

No financial conflicts exist.

\section{References}

1. Peterson L, Vasiliadis HS, Brittberg M, Lindahl A. Autologous chondrocyte implantation: a long-term follow-up. Am J Sports Med. 2010;38:11171124.

2. Pittenger MF, Mackay AM, Beck SC, et al. Multilineage potential of adult human mesenchymal stem cells. Science. 1999;284:143-147.

3. Niemeyer $\mathrm{P}$, Kornacker $\mathrm{M}$, Mehlhorn A, et al. Comparison of immunological properties of bone marrow stromal cells and adipose tissuederived stem cells before and after osteogenic differentiation in vitro. Tissue Eng. 2007;13:111-121.

4. Koob S, Torio-Padron N, Stark GB, Hannig C, Stankovic Z, Finkenzeller G. Bone formation and neovascularization mediated by mesenchymal stem cells and endothelial cells in critical-sized calvarial defects. Tissue Eng Part A. 2011;17:311-321.

5. Mehlhorn AT, Niemeyer $P$, Kaschte $K$, et al. Differential effects of BMP-2 and TGF-beta1 on chondrogenic differentiation of adipose derived stem cells. Cell Prolif. 2007;40:809-823.

6. Birmingham E, Niebur GL, McHugh PE, Shaw G, Barry FP, McNamara LM Osteogenic differentiation of mesenchymal stem cells is regulated by osteocyte and osteoblast cells in a simplified bone niche. Eur Cell Mater. 2012;23:13-27.

7. Csaki C, Matis U, Mobasheri A, Shakibaei M. Co-culture of canine mesenchymal stem cells with primary bone-derived osteoblasts promotes osteogenic differentiation. Histochem Cell Biol. 2009;131:251-266.

8. Hammoudi TM, Rivet CA, Kemp ML, Lu H, Temenoff JS. Three-dimensional in vitro tri-culture platform to investigate effects of crosstalk between mesenchymal stem cells, osteoblasts, and adipocytes. Tissue Eng Part A. 2012;18:1686-1697.

9. Schmal H, Zwingmann J, Fehrenbach $M$, et al. bFGF influences human articular chondrocyte differentiation. Cytotherapy. 2007;9:184-193.

10. Finkenzeller G, Mehlhorn AT, Schmal H, Stark GB. Post-transcriptional regulation of osteoblastic platelet-derived growth factor receptor-alpha expression by co-cultured primary endothelial cells. Cells Tissues Organs. 2010;192:28-38.

11. Loebel C, Czekanska EM, Staudacher J, et al. The calcification potential of human MSCs can be enhanced by interleukin-1beta in osteogenic medium. J Tissue Eng Regen Med. 2014. [Epub ahead of print]; DOI: 10.1002/ term.1950.

12. Kim H, Lee $\mathrm{JH}$, Suh $\mathrm{H}$. Interaction of mesenchymal stem cells and osteoblasts for in vitro osteogenesis. Yonsei Med J. 2003;44:187-197.

13. Kyllonen L, Haimi S, Mannerstrom B, et al. Effects of different serum conditions on osteogenic differentiation of human adipose stem cells in vitro. Stem Cell Res Ther. 2013;4:17.

14. Arrigoni E, Lopa S, de Girolamo L, Stanco D, Brini AT. Isolation, characterization and osteogenic differentiation of adipose-derived stem cells: from small to large animal models. Cell Tissue Res. 2009;338:401-411.

15. Kasagi S, Chen W. TGF-beta1 on osteoimmunology and the bone component cells. Cell Biosci. 2013;3:4.

16. Schagemann JC, Paul S, Casper ME, et al. Chondrogenic differentiation of bone marrow-derived mesenchymal stromal cells via biomimetic and bioactive poly-epsilon-caprolactone scaffolds. J Biomed Mater Res A. 2013;101:1620-1628.

17. Zimmermann G, Henle $P$, Kusswetter $M$, et al. TGF-beta1 as a marker of delayed fracture healing. Bone. 2005;36:779-785.

18. Weber M, Steinert A, Jor A, et al. Formation of cartilage matrix proteins by BMP-transfected murine mesenchymal stem cells encapsulated in a novel class of alginates. Biomaterials. 2002;23:2003-2013.

19. Zhu F, Friedman MS, Luo W, Woolf $P$, Hankenson KD. The transcription factor osterix (SP7) regulates BMP6-induced human osteoblast differentiation. J Cell Physiol. 2012;227:2677-2685. 
20. Aubin JE. Regulation of osteoblast formation and function. Rev Endocr Metab Disord. 2001;2:81-94.

21. Zhang J, Zhou H, Yang K, Yuan Y, Liu C. RhBMP-2-loaded calcium silicate/ calcium phosphate cement scaffold with hierarchically porous structure for enhanced bone tissue regeneration. Biomaterials. 2013;34:9381-9392.

Cite this article as: Glueck $\mathrm{M}$, Gardner $\mathrm{O}$, Czekanska $\mathrm{E}$, Alini $\mathrm{M}$, Stoddart MJ, Salzmann GM, Schma H (2015) Induction of osteogenic differentiation in human mesenchymal stem cells by crosstalk with osteoblasts. BioResearch Open Access 4:1, 121-130, DOI: 10.1089/ biores.2015.0002.

$\begin{aligned} & \text { Abbreviations Used } \\ & \mathrm{ALP}=\text { alkaline phosphatase } \\ & \mathrm{ARS}=\text { Alizarin red staining } \\ & \mathrm{CO} 1 \mathrm{~A} 1=\text { collagen type } 1 \\ & \mathrm{DMEM}=\text { Dulbecco's modified Eagle's medium } \\ & \mathrm{FBS}=\text { fetal bovine serum } \\ & \mathrm{MSC}=\text { mesenchymal stem cells } \\ & \mathrm{OB}=\text { human osteoblasts } \\ & \mathrm{OD}=\text { optical density } \\ & \mathrm{PDR}=\text { population doubling rate } \\ & \mathrm{TGF} \beta=\text { transforming growth factor beta }\end{aligned}$

\section{Publish in BioResearch Open Access}

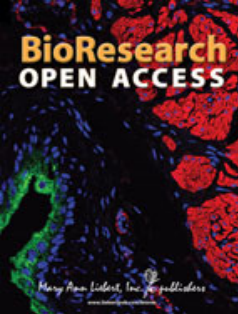

- Broad coverage of biomedical research - Immediate, unrestricted online access

- Rigorous peer review

- Compliance with open access mandates

- Authors retain copyright

- Highly indexed

- Targeted email marketing

liebertpub.com/biores 